\title{
DHT bypasses testosterone to drive progression to castration resistance
}

Progression of prostate cancer towards castration resistance mainly involves the synthesis of dihydrotestosterone (DHT) from $5 a$-dione, instead of via testosterone.

DHT is the principal androgen bound to the androgen receptor in prostate cancer cell nuclei. It was previously believed that DHT was synthesized from androstenedione via testosterone, implying that testosterone was the obligate precursor of DHT. However, a team from the University of Texas Southwestern Medical Center have used prostate cancer cell lines, human tissue samples and mouse xenograft models to identify a novel synthetic pathway through which androgens are produced in tumor cells.

Using cell lines derived from six patients with castration-resistant prostate cancer (CRPC), the team showed that conversion of androstenedione to $5 a$-dione occurred earlier and more rapidly than that of androstenedione to testosterone. Incubation of fresh tissue from metastatic prostate cancer biopsies with labeled androstenedione and testosterone confirmed these results, showing that flux from androstenedione to $5 \alpha$-dione was greater than that of testosterone to DHT, and suggesting that the dominant route of androstenedione metabolism is via $5 a$-dione. Furthermore, when LNCaP xenograft tumors were implanted in orchidectomized mice, supplementation with androstenedione resulted in faster tumor growth and progression than testosterone supplementation.

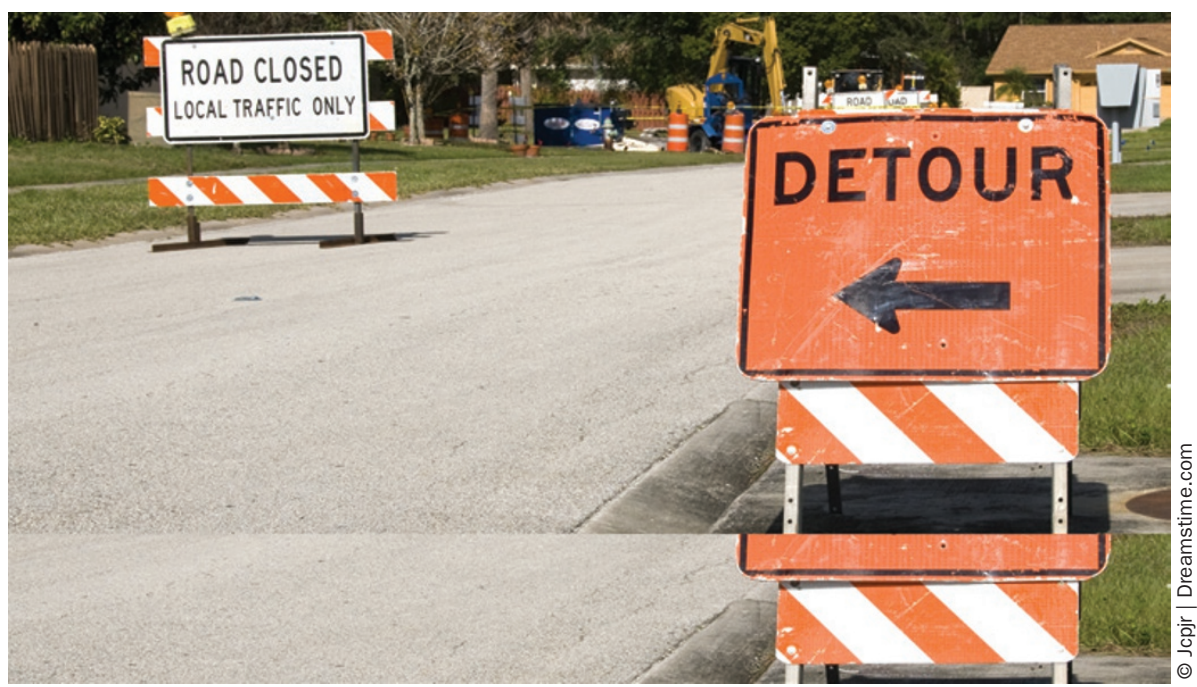

The authors also investigated the enzymes involved in this synthetic pathway. Expression of the SRD5A1 enzyme is upregulated during progression to CRPC, but the SRD5A2 isoform is downregulated. The team used lentiviral short-hairpin RNAs (shRNAs) to knock down SRD5A1 and SRD5A2 expression in prostate cancer cell lines. Knock down of SRD5A1 almost ablated the metabolism of androstenedione in cultured cells, with a blockade of $5 \alpha$-dione and DHT synthesis. However, the conversion of androstenedione to testosterone was increased. This effect was not seen when SRD5A2 was knocked down, suggesting that this isoform is not involved in the alternative pathway. Taking into account the expression changes seen in these enzymes in CRPC, these data indicate that prostate cancer progression involves the selection of tumor cells that can synthesize DHT via the alternative pathway, escaping the effect of drugs such as dutasteride, which inhibit the SRD5A enzymes.

These results signify a paradigm shift in our understanding of androgen synthesis, and future studies regarding the progression to CRPC will need to consider the role of this novel pathway.

\section{Annette Fenner}

Original article Chang, K. H. et al. Dihydrotestosterone synthesis bypasses testosterone to drive castrationresistant prostate cancer. Proc. Natl Acad. Sci. USA doi:10.1073/pnas.1107898108 